Pacific Journal of Mathematics

IMAGINARY VALUES OF MEROMORPHIC FUNCTIONS IN 


\section{IMAGINARY VALUES OF MEROMORPHIC FUNCTIONS IN THE DISK}

Douglas W. TOWNSEND

Let $f$ be a meromorphic function in the unit disk, and let $\phi(r, f)$ be the number of solutions of the equation $\operatorname{Re} f\left(r e^{i \theta}\right)=0$ for $0 \leqq \theta \leqq 2 \pi$. In this paper we bound $\phi(r, f)$ off an exceptional set of $r$ values, and $\Phi(r, f)=\int_{0}^{r} \phi(t, f)(1-t)^{-1} d t$ for all $r$, in terms of the Nevanlinna characteristic function of $f$. We then give examples to show that the bounds obtained are the best possible.

The quantity $\phi(r, f)$ was studied for entire functions by $\mathrm{A}$. Gelfond [3] and later by S. Hellerstein and J. Korevaar [5]. The quantities $\phi(r, f)$ and $\Phi(r, f)$ were studied for meromorphic functions in the plane by J. Miles and the author [10].

We will prove the following theorem analogous to Theorem 1 of Miles and Townsend.

THeOREM. If $c_{0}(r)=\left(1-\alpha_{0}\right)+\alpha_{0} r$ for $0<\alpha_{0}<1$ and $f$ is a meromorphic function in the unit disk then there is a constant $A=A\left(\alpha_{0}\right)$ and $a$ set $\Delta \subset[0,1)$ satisfying

$$
\int_{\Delta} \exp \left\{T\left(c_{0}(r), f\right)-\log (1-r)\right\} d r<\infty
$$

so that for $r \notin \Delta$ and $r>R$

(i) $\dot{\phi}(r, f)<A(1-r)^{-1}\left[T\left(c_{0}(r), f\right)-\log (1-r)\right]$. If $\Phi(r, f)=\int_{0}^{r} \phi(t, f)(1-t)^{-1} d t$ then there is an $\alpha_{1}$ so that $0<\alpha_{1}<1$, and a constant $A^{\prime}$ so that for $r>R$ and for $c_{1}(r)=\left(1-\alpha_{1}\right)+\alpha_{1} r$

(ii ) $\Phi(r, f)<A^{\prime}(1-r)^{-1}\left[T\left(c_{1}(r), f\right)+(1-r)^{-1}\right]$.

We will then give examples to show that no nontrivial lower bound for $\phi(r, f)$ can be given and that the factor $(1-r)^{-1}$ in (i) and (ii) can not be replaced by any function $b(r)$ satisfying $b(r)=$ $o\left((1-r)^{-1}\right)$ as $r \rightarrow 1$.

It is not known whether the exceptional set for (i) is nonempty, even if $f$ is holomorphic in the unit disk.

We note that the second occurrence of $(1-r)^{-1}$ in (ii) may be replaced by $-\log (1-r)$, using a proof that is much longer and more intricate than the one given in this paper. This alternate proof is a combination of the essential ideas of the proof of Theorem 2 in [12], together with techniques used in this paper to bound $\phi(r, f)$ in terms of the characteristic function of $f$. 
The technique used in [10] to obtain an upper bound for the number of solutions of $\operatorname{Re} g(z)=0$ on $|z|=r$ for $g$ meromorphic in the plane begins by considering $G_{r}(\theta)=\operatorname{Re} g\left(r e^{i \theta}\right)$ as a function of a complex variable $\theta$. After showing that $G_{r}(\theta)$ is a meromorphic function in the $\theta$-plane, Jensen's theorem can be used to bound the number of zeros of $G_{r}$ in $|\theta| \leqq \pi$, and hence to bound the number of zeros of $\operatorname{Re} g\left(r e^{i \theta}\right)$ for $-\pi \leqq \theta \leqq \pi$. However, if $g$ is meromorphic in $|z|<1$, then $G_{r}(\theta)$ is only meromorphic in $|\operatorname{Im} \theta|<A(1-r)$, where $0<A<1$. Thus, to bound the number of zeros of $G_{r}(\theta)$ on the real $\theta$-axis using the above technique, we would have to apply Jensen's theorem to $G_{r}(\theta)$ in $O\left((1-r)^{-1}\right)$ disks of radius less than $A(1-r)$, centered on the real $\theta$-axis, and covering the real $\theta$-axis between $-\pi$ and $\pi$. This complication alone would introduce an additional factor of $(1-r)^{-1}$ to the bounds of $\phi$ and $\Phi$ in (i) and (ii) of the theorem. New techniques are used to obtain the correct bounds for $\phi$ and $\Phi$.

Also, in [10] the bounds on $\phi$ and $\Phi$ involve $T(A r, f)$ for some constant $A>1$. Such a bound is impossible for $r$ close to 1 if $f$ is meromorphic in $|z|<1$. This complication is resolved by denoting a convex linear combination of 1 and $r$ by $c(r)=(1-b)+b r, 0<$ $b<1$, and bounding $\dot{\phi}$ and $\Phi$ in terms of $T(c(r), f){ }^{1}$

We assume familiarity with the standard notation of Nevanlinna theory. It is not intended that positive constants such as $A$ and $R$ have the same value with each occurrence. Also, notation such as $A\left(\alpha_{0}\right), A(\alpha, d)$, etc. is used to emphasize the dependence of the constants on $\alpha_{0}$, or $\alpha$ and $d$, etc. Once again it is not intended that these constants have the same value with each occurrence. Throughout the paper, if $c(r)=(1-b)+b r$ for $0<b<1$, then we let $c^{n}(r)=c\left(c^{n-1}(r)\right)$. It is easy to show that $c^{n}(r)=\left(1-b^{n}\right)+b^{n} r$.

\section{Preliminary lemmas.}

LEMma 1.1. ${ }^{2}$ Let $f(z)$ be holomorphic in the circle $|\boldsymbol{z}|<R$ with ${ }_{1} f(0) \mid=1$ and let $\eta$ be an arbitrary positive number not exceeding $(8 e)^{-1}$. Inside the circle $|z| \leqq r<R$ but outside of a family of excluded circles, centered at the zeros of $f$ in $|z|<R$, the sum of whose radii is not greater than $\eta r$, we have

$$
\log |f(z)|>A(R-r)^{-2} T(R, f) \log \eta,
$$

provided $r$ and $R$ are sufficiently large.

${ }^{1}$ I wish to thank the referee of this paper for suggesting this very useful notation as well as for making other helpful comments.

2 This lemma was observed several years ago by A. Baernstein, who in unpublished work used it to obtain a bound for $\phi(r, f)$, off an exceptional set, where $f$ is meromorphic in the plane. 
This is an elementary adaptation of Theorem 11 of [7].

Lemma 1.2. There are absolute constants $A>0, \gamma \in[0,1)$ and $p$, a positive integer, such that if $f$ is meromorphic in $|z|<1$, then there exist holomorphic functions $g$ and $h$ in $|z|<1$, such that $f=$ $g / h$ and

$$
\max (T(r, g), T(r, h))<A(1-r)^{-p} T((1-\gamma)+\gamma r, f) .
$$

This lemma is contained in [1], which carries a result of J. Miles [9] to the unit disk.

LEMMA 1.3. If $f$ is a nonconstant meromorphic function in the plane and $0<\alpha<1$, then there is an $A=A(\alpha)$ so that for $r>R$

$$
\begin{aligned}
\frac{1}{2 \pi} \int_{0}^{2 \pi}\left|\operatorname{Re}\left(r e^{i \theta} f^{\prime \prime}\left(r e^{i \theta}\right) / f^{\prime}\left(r e^{i \theta}\right)\right)+1\right| d \theta \\
\quad<A(1-r)^{-1}[T((1-\alpha)+\alpha r, f)-\log (1-r)] .
\end{aligned}
$$

This lemma is contained in (3.10) of [8].

LEMMA 1.4. Suppose $f$ is a nonconstant meromorphic function in the disk and $r$ is such that $f^{\prime}\left(r e^{i \theta}\right) \neq 0, \infty$ for $0 \leqq \theta \leqq 2 \pi$. If $\dot{\phi}(r, f)>7 A(1-r)^{-1}[T((1-\alpha)+\alpha r, f)-\log (1-r)]$, where $A$ and $\alpha$ are as in Lemma 1.3, then

$$
\phi\left(r, z f^{\prime \prime}(z) / f^{\prime}(z)+1\right)>\phi(r, f) / 6 .
$$

Proof. Let $\beta(\theta)$ be a continuous determination of the argument of the vector tangent to the curve $f\left(r e^{i \theta}\right), 0 \leqq \theta \leqq 2 \pi$. We recall that

$$
\beta^{\prime}(\theta)=\operatorname{Re}\left(r e^{i \theta} f^{\prime \prime}\left(r e^{i \theta}\right) / f^{\prime}\left(r e^{i \theta}\right)+1\right) .
$$

Suppose $0 \leqq \alpha_{1}<\alpha_{2}<\alpha_{3}<2 \pi, \quad \operatorname{Re} f\left(r e^{i \alpha_{j}}\right)=0$ for $j=1,2,3$ and $\operatorname{Re} f\left(r e^{i \theta}\right) \neq 0$ for $\alpha_{1}<\theta<\alpha_{3}$ except for $\theta=\alpha_{2}$. We distinguish two cases.

Case I. Suppose $\left|\beta\left(\dot{\phi}_{1}\right)-\beta\left(\dot{\phi}_{2}\right)\right|<\pi$ for all $\phi_{1}$ and $\dot{\phi}_{2}$ in $\left[\alpha_{1}, \alpha_{3}\right]$. By Rolle's theorem there exist $\alpha_{1}^{\prime} \in\left(\alpha_{1}, \alpha_{2}\right)$ and $\alpha_{2}^{\prime} \in\left(\alpha_{2}, \alpha_{3}\right)$ and there exist integers $n_{1}$ and $n_{2}$ such that $\beta\left(\alpha_{j}^{\prime}\right)=n_{j} \pi+\pi / 2, j=1,2$. Since $\left|\beta\left(\alpha_{1}^{\prime}\right)-\beta\left(\alpha_{2}^{\prime}\right)\right|<\pi$, we must have $\beta\left(\alpha_{1}^{\prime}\right)=\beta\left(\alpha_{2}^{\prime}\right)$. By Rolle's theorem we conclude that in Case I there exists $\gamma$ in $\left(\alpha_{1}^{\prime}, \alpha_{2}^{\prime}\right) \subset\left(\alpha_{1}, \alpha_{3}\right)$ such that $\beta^{\prime}(\gamma)=0$.

Case II. Suppose there exist $\dot{\phi}_{1}$ and $\phi_{2}$ in $\left[\alpha_{1}, \alpha_{2}\right]$ such that $\left|\beta\left(\dot{\phi}_{1}\right)-\beta\left(\dot{\phi}_{2}\right)\right| \geqq \pi$. Thus, in Case II 


$$
\frac{1}{2 \pi} \int_{\alpha_{1}}^{\alpha_{3}}\left|\beta^{\prime}(\theta)\right| d \theta \geqq \frac{1}{2} .
$$

We now let $0 \leqq \theta_{1}<\theta_{2}<\cdots<\theta_{n}<2 \pi$ be a complete list of solutions of $\operatorname{Re} f\left(r e^{i \theta}\right)=0$ in $[0,2 \pi)$, and consider triples $\left(\theta_{2 k-1}, \theta_{2 k}, \theta_{2 k+1}\right)$ for $1 \leqq k \leqq[\phi(r, f) / 2]-1$. By Lemma 1.3 and (1.2), no more than $2 A(1-r)^{-1}[T((1-\alpha)+\alpha r, f)-\log (1-r)]$ of these triples fall into Case II. Thus at least

$$
\begin{aligned}
{[\phi(r, f) / 2]-} & 1-\left[2 A(1-r)^{-1}\{T((1-\alpha)+\alpha r, f)-\log (1-r)\}\right] \\
& \geqq[\phi(r, f) / 6]
\end{aligned}
$$

of these triples fall into Case I, and consequently there are at least $\phi(r, f) / 6$ zeros of $\beta^{\prime}(\theta)$ in $[0,2 \pi)$.

LEMMA 1.5. If $f$ is a nonconstant meromorphic function in the unit disk, $k(r)$ is a function satisfying $k(r) \geqq-\log (1-r)$ and $c_{2}(r)=\left(1-\alpha_{2}\right)+\alpha_{2} r$ where $0<\alpha_{2}<1$, then there is a constant $A$ and $a$ set $\Delta \subset[0,1)$, both depending on the function $k$ and on $\alpha_{2}$, such that

$$
\int_{\Delta} \exp \left\{T\left(c_{2}(r), f\right)+k(r)\right\} d r<\infty
$$

and for $r \notin \Delta$ and $r>R$,

$$
\int_{0}^{2 \pi} \log \left|\operatorname{Re}\left(r e^{i \theta} f^{\prime \prime}\left(r e^{i \theta}\right) / f^{\prime}\left(r e^{i \theta}\right)\right)+1\right|^{-1} d \theta<A\left[T\left(c_{2}(r), f\right)+k(r)\right] .
$$

Proof. We follow closely [6, p. 226-227]. Let $G(z)=z f^{\prime \prime}(z) / f^{\prime}(z)+1$, and

$$
\rho(a)=|\operatorname{Re} a|^{-1 / 2}\left(\iint_{A}|\operatorname{Re} a|^{-1 / 2} d w(a)\right)^{-1}
$$

where $w(a)$ is area measure on the Riemann sphere $A$. Also, define

$$
\lambda(t, G)=\int_{0}^{2 \pi} \rho\left(G\left(t e^{i \theta}\right)\right)\left|G^{\prime}\left(t e^{i \theta}\right)\right|^{2}\left(1+\left|G\left(t e^{i \theta}\right)\right|^{2}\right)^{-2} d \theta .
$$

From (14.6.18) of [6], we have

$$
\int_{0}^{2 \pi} \log \rho\left(G\left(r e^{i \theta}\right)\right) d \theta \leqq 8 \pi T(r, G)+\log \lambda(r, G)+O(1) .
$$

We set $L(r, G)=\int_{0}^{r} \lambda(t, G) t d t$ and $K(r, G)=\int_{r_{0}}^{r} L(s, G) s^{-1} d s$. Then by (14.6.20) of [6], $T(r, G) \geqq K(r, G)-O(1)$. Denote by $\Delta_{1}$ the intervals $\left(\alpha_{1 j}, \beta_{1 j}\right)$ where

$$
\lambda(r, G)>r^{-1} \exp \left\{k(r)+T\left(c_{2}(r), G\right)\right\}(L(r, G))^{2} .
$$


We have

$$
\begin{aligned}
\int_{\Delta_{1}} \exp \left\{k(r)+T\left(c_{2}(r), G\right)\right\} d r & <\int_{\Delta_{1}} r \lambda(r, G)(L(r, G))^{-2} d r \\
& =\int_{\Delta_{1}}(L(r, G))^{-2} d L(r, G) \\
& <\left(L\left(\alpha_{11}, G\right)\right)^{-1}<\infty .
\end{aligned}
$$

Denote by $\Delta_{2}$ the intervals $\left(\alpha_{2 j}, \beta_{2 j}\right)$ where

$$
L(r, G)>r \exp \left\{k(r)+T\left(c_{2}(r), G\right)\right\}[K(r, G)]^{2} .
$$

As before, we have

$$
\begin{aligned}
\int_{\Delta_{2}} \exp \left\{k(r)+T\left(c_{2}(r), G\right)\right\} d r & <\int_{\Delta_{2}}(K(r, G))^{-2} d(K(r, G)) \\
& <\left(K\left(\alpha_{21}, G\right)\right)^{-1}<\infty
\end{aligned}
$$

Let $\Delta=\Delta_{1} \cup \Delta_{2}$. If $r \notin \Delta$ and $r>R$, then

$$
\begin{aligned}
\lambda(r, G) & <r^{-1} \exp \left\{k(r)+T\left(c_{2}(r), G\right)\right\}[L(r, G)]^{2} \\
& <r \exp \left\{3 k(r)+3 T\left(c_{2}(r), G\right)\right\}(K(r, G))^{4} \\
& <r \exp \left\{3 k(r)+3 T\left(c_{2}(r), G\right)\right\}(T(r, G)+O(1))^{4} .
\end{aligned}
$$

Thus for $r \notin \Delta$ and $r>R$ and for some constant $A$,

$$
\log \lambda(r, G)<A\left(3 k(r)+7 T\left(c_{2}(r), G\right)\right) .
$$

From Lemma 1.6 and well known properties of the characteristic function, $T(s, G)<A_{2}(T(s, f)-\log (1-s))$ for $s>R$. The lemma follows readily from (1.3) and (1.4).

We state the following elementary lemma without proof.

LEMMA 1.6. Let $f$ be meromorphic in $|z|<1$ with $|f(0)|=1$. If $r<1$ and $c(r)=(1-\alpha)+\alpha r$ for some $0<\alpha<1$, then

(i ) $n\left(r, f^{\prime}\right)<A(\alpha)(1-r)^{-1} T\left(c(r), f^{\prime}\right)$

(ii) $n\left(r, 1 / f^{\prime}\right)<A(\alpha)(1-r)^{-1} T\left(c(r), f^{\prime}\right)$

(iii) $T\left(r, f^{\prime}\right)<A(T(r, f)-\log (1-r))$ for $r>R$

and

(iv) $T\left(r, 1 / f^{\prime}\right)<A(T(r, f)-\log (1-r))$ for $r>R$.

2. Proof of part (i) of the theorem. Without loss of generality we may assume that $|f(0)|=1$ since if $f(0) \neq 0$, $\infty$ we may consider $f(z) /|f(0)|$ and if $f(0)=0$, $\infty$ we may consider $f(z)+i$ or $1 / f(z)+i$.

With $\alpha_{0}$ as in part (i) of the theorem, let

$$
\alpha=\alpha_{0}^{1 / 2} \text { and } s=c(r)=(1-\alpha)+\alpha r .
$$

Also define 


$$
F_{r}(\theta)=\operatorname{Re}\left(r e^{i \theta} f^{\prime \prime}\left(r e^{i \theta}\right) / f^{\prime}\left(r e^{i \theta}\right)\right)+1,
$$

and for $x \in[0,2 \pi)$

$$
H_{r}^{x}(\theta)=F_{r}(x+\theta) .
$$

We will show that if $\theta$ is complex then $H_{r}^{x}(\theta)$ is a meromorphic function in a strip containing the real $\theta$-axis. We will apply Jensen's theorem to $H_{r}^{x}(\theta)$ in a circle centered on the real $\theta$-axis, and integrate with respect to $x$ to obtain a bound for $\phi\left(r,\left(z f^{\prime}(z) / f(z)\right)+1\right)$, which will yield a bound for $\phi(r, f)$. We first let

$$
K(t, a, \theta)=\left(t^{2}-t a \cos \theta\right) /\left(t^{2}+a^{2}-2 a t \cos \theta\right) .
$$

Then, by the differentiated Poisson-Jensen theorem [4, p. 22], we have

$$
\begin{aligned}
F_{r}(\theta)= & \frac{1}{2 \pi} \int_{0}^{2 \pi} \log \left|f^{\prime}\left(s e^{i \mu}\right)\right| \frac{2 r s\left(\left(r^{2}+s^{2}\right) \cos (\theta-\mu)-2 r s\right)}{\left(s^{2}+r^{2}-2 r s \cos (\theta-\mu)\right)^{2}} d \mu \\
& -\sum_{0<a_{n}<s} K\left(a_{n} r, s^{2}, \theta-\alpha_{n}\right)+\sum_{0<b_{n}<s} K\left(b_{n} r, s^{2}, \theta-\beta_{n}\right) \\
& +\sum_{a_{n}<s} K\left(r, a_{n}, \theta-\alpha_{n}\right)-\sum_{b_{n}<s} K\left(r, b_{n}, \theta-\beta_{n}\right)+1 \\
= & I-I I+I I I+I V-V+1,
\end{aligned}
$$

where $\left\{a_{n} e^{i \alpha_{n}}\right\}$ and $\left\{b_{n} e^{i \beta_{n}}\right\}$ are the zeros and poles, respectively, of $f^{\prime}$, listed in nondecreasing order of magnitude. We let $\theta$ be complex and prove

LEMma 2.1. The function $F_{r}(\theta)$ (see (2.1)) is meromorphic in $|\operatorname{Im} \theta|<(1-\alpha)(1-r)$ with poles at values of $\theta$ for which $\operatorname{Im} \theta=$ $\pm \log \left(r d_{n}^{-1}\right)$ and $\operatorname{Re} \theta=\gamma_{n}+2 \pi k, k=0, \pm 1, \pm 2, \cdots$, where $d_{n} e^{i_{i n}}$ is a zero or pole of $f^{\prime}$ and $0<d_{n}<s$.

Proof. If $t=a$ then $K(t, a, \theta)=1 / 2$ for all $\theta \neq 2 \pi k, k=0, \pm 1$, $\pm 2, \cdots$. If $t^{2}+a^{2}-2 a t \cos \theta=0$ where $a \neq t$ and $\theta=\zeta+i \beta$, then

$$
1<\left(a^{2}+t^{2}\right)(2 a t)^{-1}=\cos \theta=\cos \zeta \cosh \beta-i \sin \zeta \sinh \beta .
$$

Thus, $\zeta=2 \pi k$ and $\cosh \beta=\left(a^{2}+t^{2}\right) / 2 a t=(a / t+t / a) / 2=\cosh (\log a / t)$. Hence,

$$
\operatorname{Re} \theta=2 \pi k, \quad k \text { an integer and } \operatorname{Im} \theta= \pm \log a t^{-1} .
$$

We have $\log s r^{-1}=\log \left(1+(s-r) r^{-1}\right)>(1-\alpha)(1-r)$ for $r>R$. Thus, term $I$ of (2.2) is a holomorphic function of $\theta$ in $|\operatorname{Im} \theta|<$ $(1-\alpha)(1-r)$. Also for $0<d_{n}<s$, we have $\log s^{2}\left(d_{n} r\right)^{-1}>\log s r^{-1}$. Hence terms $I I$ and $I I I$ are also holomorphic in $|\operatorname{Im} \theta|<(1-\alpha)(1-r)$. Finally, from (2.5) and (2.6), terms $I V$ and $V$ are meromorphic in $|\operatorname{Im} \theta|<(1-\alpha)(1-r)$ with poles at values of $\theta$ satisfying $\operatorname{Im} \theta=$ 
$\pm \log r d_{n}^{-1}$ and $\operatorname{Re} \theta=\gamma_{n}+2 \pi k, k=0, \pm 1, \pm 2, \cdots$.

We now apply Jensen's theorem to $H_{r}^{x}(\theta)$ (see (2.2)) with $h=$ $(1-\alpha)(1-r) / 2$, and integrate with respect to $x$, to obtain

$$
\begin{aligned}
\int_{0}^{2 \pi} N\left(h, \frac{1}{H_{r}^{x}}\right) d x= & -\int_{0}^{2 \pi} \log \left|H_{r}^{x}(0)\right| d x+\int_{0}^{2 \pi} N\left(h, H_{r}^{x}\right) d x \\
& +\int_{0}^{2 \pi} \frac{1}{2 \pi} \int_{0}^{2 \pi} \log \left|H_{r}^{x}\left(h e^{i \mu}\right)\right| d \mu \\
= & L_{1}+L_{2}+L_{3} .
\end{aligned}
$$

In the following four lemmas we obtain a lower bound for the left hand side of equation (2.7), and upper bounds for the three terms $L_{1}, L_{2}$ and $L_{3}$.

LEMMA 2.2. For $H_{r}^{x}$ defined above we have

$$
\int_{0}^{2 \pi} N\left(h, \frac{1}{H_{r}^{x}}\right) d x \geqq 2 h \phi\left(r, z f^{\prime \prime}(z) / f^{\prime}(z)+1\right) .
$$

Proof. By Tonelli's theorem,

$$
\int_{0}^{2 \pi} N\left(h, \frac{1}{H_{r}^{x}}\right) d x=\int_{0}^{h} \int_{0}^{2 \pi} n\left(t, \frac{1}{H_{r}^{x}}\right) t^{-1} d x d t .
$$

The contribution to the latter integral from a single zero of $H_{r}^{x}$ on the real $\theta$-axis at $\theta=a$, where $0 \leqq a-h<a+h<2 \pi$ is $\int_{0}^{h} \int_{a-t}^{a+t} t^{-1} d x d t=$ $2 \int_{0}^{h} d t=2 h$. Similarly it can be shown that if $a-h<0$ or $a+h \geqq$ $2 \pi$, then the contribution to the integral is again $2 h$. The lemma follows from the fact that the real zeros of $H_{r}^{x}$ are just the zeros of $\operatorname{Re}\left(z f^{\prime \prime}(z) / f^{\prime}(z)+1\right)$ on $|z|=r$.

Lemma 2.3. Let $A$ be the constant and $\Delta$ the set in Lemma 1.5 corresponding to $k(r)=-\log (1-r)$ and $\alpha_{2}=\alpha^{2}$. For $L_{1}$ as in (2.7) we have for $r \notin \Delta$ and $r>R$,

$$
L_{1}<A\left[T\left(c^{2}(r), f\right)-\log (1-r)\right] .
$$

Proof. If $r \notin \Delta$ and $r>R$, then by Lemma 1.5

$$
\begin{aligned}
L_{1} & =-\int_{0}^{2 \pi} \log \left|H_{r}^{x}(0)\right| d x=-\int_{0}^{2 \pi} \log \left|F_{r}(x)\right| d x \\
& =-\int_{0}^{2 \pi} \log \left|\operatorname{Re}\left(r e^{i x} f^{\prime \prime}\left(r e^{i x}\right) / f^{\prime}\left(r e^{i x}\right)\right)+1\right| d x \\
& <A\left[T\left(c^{2}(r), f\right)-\log (1-r)\right] .
\end{aligned}
$$

LEMmA 2.4. For $L_{2}$ as in (2.7), we have for $A=A(\alpha)$ and for 
$r>R$

$$
L_{2}<A\left[T\left(c^{2}(r), f\right)-\log (1-r)\right] \text {. }
$$

Proof. By Tonelli's theorem we have

$$
L_{2}=\int_{0}^{2 \pi} N\left(h, H_{r}^{x}\right) d x=\int_{0}^{h} \int_{0}^{2 \pi} n\left(t, H_{r}^{x}\right) t^{-1} d x d t .
$$

The contribution to $L_{2}$ from a pole of $F_{r}(\theta)$ at $b$, where $|\operatorname{Im} b|<h$, is no more than

$$
\begin{aligned}
\int_{|\operatorname{Im} b|}^{h}\left(\int_{\operatorname{Re} b-\sqrt{t} 2-(\operatorname{Im} b)^{2}}^{\operatorname{Re} b+\sqrt{t^{2}-(\operatorname{Im} b)^{2}}} d x\right) t^{-1} d t & =\int_{|\operatorname{Im} b|}^{h} 2 \sqrt{t^{2}-(\operatorname{Im} b)^{2}} t^{-1} d t \\
& \leqq 2 \int^{h} d t=2 h
\end{aligned}
$$

The poles of $F_{r}(\theta)$ (see (2.1)) in $\{\theta: 0 \leqq \operatorname{Re} \theta<2 \pi$ and $|\operatorname{Im} \theta|<h\}$ arise from zeros or poles of $f^{\prime}(z)$ in $|z|<s$. Thus, by Lemma 1.6, $F_{r}(\theta)$ has no more than $2\left(n\left(s, f^{\prime}\right)+n\left(s, 1 / f^{\prime}\right)\right)<A(\alpha)(1-r)^{-1}\left[T\left(c^{2}(r), f\right)-\right.$ $\log (1-r)]$ poles in the above region for $r>R$. Hence

$$
L_{2}<2 h A(\alpha)(1-r)^{-1}\left[T\left(c^{2}(r), f\right)-\log (1-r)\right],
$$

and the lemma follows since $h(1-r)^{-1}=(1-\alpha) / 2$.

LEMMA 2.5. For $L_{3}$ as in (2.7) we have for some constant $A=$ $A(\alpha)$ and for $r>R$

$$
L_{3}<A\left[T\left(c^{2}(r), f\right)-\log (1-r)\right] .
$$

Proof. We have from (2.4) that

$$
\begin{aligned}
L_{3}= & \frac{1}{2 \pi} \int_{0}^{2 \pi} \int_{0}^{2 \pi} \log \left|F_{r}\left(x+h e^{i \mu}\right)\right| d x d \mu \\
\leqq & \frac{1}{2 \pi} \int_{0}^{2 \pi} \int_{0}^{2 \pi} \log ^{+}\left|\frac{1}{2 \pi} \int_{0}^{2 \pi} \log \right| f^{\prime}\left(s e^{i t}\right) \mid \\
& \times \frac{2 r s\left(\left(s^{2}+r^{2}\right) \cos \left(x+h e^{i \mu}-t\right)-2 r s\right)}{\left(r^{2}+s^{2}-2 r s \cos \left(x+h e^{i \mu}-t\right)\right)^{2}} d t \mid d x d \mu \\
& +\frac{1}{2 \pi} \int_{0}^{2 \pi} \int_{0}^{2 \pi} \log ^{+}\left|\sum_{d_{n}<s} K\left(r, d_{n}, x+h e^{i \mu}-\gamma_{n}\right)\right| d x d \mu \\
& +\frac{1}{2 \pi} \int_{0}^{2 \pi} \int_{0}^{2 \pi} \log ^{+}\left|\sum_{0<d_{n}<s} K\left(d_{n} r, s^{2}, x+h e^{i \mu}-\gamma_{n}\right)\right| d x d \mu+\log 5 \\
= & E_{1}+E_{2}+E_{3}+\log 5,
\end{aligned}
$$

where $d_{n} e^{i_{\gamma_{n}}}$ is a zero or pole of $f^{\prime}$.

We analyze terms $E_{1}, E_{2}$ and $E_{3}$ separately.

Term $E_{1}$. Since $h=(1-\alpha)(1-r) / 2$ and $\log s r^{-1}>(1-\alpha)(1-r)$ 
for $r>R$, we have for some $w \in((1-\alpha)(1-r) / 2,(1-\alpha)(1-r))$, for $\mu \in[0,2 \pi)$ and for $r>R$,

$$
\begin{aligned}
\mid\left(r^{2}+\right. & \left.s^{2}\right)(2 r s)^{-1}-\cos \left(x+h e^{i \mu}-t\right) \mid \\
& \geqq\left|\cosh \left(\log s r^{-1}\right)-\cosh (h \sin \mu)\right| \\
& \geqq\left|\cosh ((1-\alpha)(1-r))-\cosh \left(\frac{1}{2}(1-\alpha)(1-r)\right)\right| \\
& =\sinh \omega\left((1-\alpha)(1-r)-\frac{1}{2}(1-\alpha)(1-r)\right) \\
& \geqq \frac{1}{2}(1-\alpha)(1-r) \sinh \left(\frac{1}{2}(1-\alpha)(1-r)\right) \\
& \geqq \frac{1}{2}(1-\alpha)(1-r) \frac{1}{4}(1-\alpha)(1-r)=\frac{1}{8}(1-\alpha)^{2}(1-r)^{2}
\end{aligned}
$$

Also, since $r<s<1$ and $\cosh (h)+\sinh (h)=e^{h}<4$, we have from (2.5) that

$$
\left|\left(s^{2}+r^{2}\right) \cos \left(x+h e_{i}{ }^{\mu}-t\right)-2 r s\right| \leqq 2(\cosh (h)+\sinh (h))+2<10 .
$$

Thus, for constants $A_{j}=A_{j}(\alpha), j=1,2$, and for $r>R$, from (2.7) and Lemma 1.6,

$$
\begin{aligned}
E_{1} & <2 \pi\left(-A_{1} \log (1-r)+\log ^{+}\left|\frac{1}{2 \pi} \int_{0}^{2 \pi}\right| \log \left|f^{\prime}\left(s e^{i t}\right)\right||d t|\right) \\
& =2 \pi\left(-A_{1} \log (1-r)+\log ^{+}\left(T\left(s, f^{\prime}\right)+T\left(s, \frac{1}{f^{\prime}}\right)\right)\right) \\
& <A_{2}(\log T(c(s), f)-\log (1-r)) .
\end{aligned}
$$

Term $E_{3}$. Since $0<d_{n}<s$ we have $\left(s^{4}+d_{n}^{2} r^{2}\right)\left(2 d_{n} r s^{2}\right)^{-1} \geqq\left(s^{2}+\right.$ $\left.r^{2}\right)(2 r s)^{-1}$. As in (2.9) we have for $r>R$ that the denominator of $\left|K\left(d_{n} r, s^{2}, x+h e^{i \mu}-\gamma_{n}\right)\right|$ (see (2.3)) divided by $\left|2 d_{n} r s^{2}\right|$ is

$$
\left|\left(s^{4}+d_{n}^{2} r^{2}\right)\left(2 d_{n} r s^{2}\right)^{-1}-\cos \left(x+h e^{i \mu}-\gamma_{n}\right)\right|>\frac{1}{8}(1-\alpha)^{2}(1-r)^{2} .
$$

Also as above we have for $r>R$ and $d_{n} \neq 0$ that the numerator of $\left|K\left(d_{n} r, s^{2}, x+h e^{i \mu}-\gamma_{n}\right)\right|$ divided by $\left|2 d_{n} r s^{2}\right|$ is

$$
\begin{aligned}
\mid\left(2 d_{n} r s^{2}\right)^{-1}\left(d_{n} r\right. & \left.s^{2} \cos \left(x+h e^{i \mu}-\gamma_{n}\right)-d_{n}^{2} r^{2}\right) \mid \\
& =\frac{1}{2}\left|\cos \left(x+h e^{i \mu}-\gamma_{n}\right)-d_{n} r s^{-2}\right| \\
& \leqq \frac{1}{2}(\cosh (h)+\sinh (h))+\frac{1}{2} \\
& =\frac{1}{2}\left(e^{h}+1\right)<3 .
\end{aligned}
$$


We conclude from (2.11) and (2.12) that for $r>R$

$$
\left|K\left(d_{n} r, s^{2}, x+h e^{i \mu}-\gamma_{n}\right)\right|<A(\alpha)(1-r)^{-2},
$$

and therefore from (2.8) and Lemma 1.6, for $r>R$

$$
\begin{aligned}
E_{3} & <2 \pi\left(\log \left(n\left(s, f^{\prime}\right)+n\left(s, 1 / f^{\prime}\right)\right)+\log \left(A(\alpha)(1-r)^{-2}\right)\right) \\
& <A(\alpha)\left[\log T\left(c^{2}(r), f\right)-\log (1-r)\right] .
\end{aligned}
$$

Term $E_{2}$. We change the variables of integration in $E_{2}$ to $u=$ $x+h \cos \mu-\gamma_{n}$ and $v=h \sin \mu$. Since this transformation takes $\{(x, \mu): 0 \leqq x<2 \pi, 0 \leqq \mu<2 \pi\}$ onto $\{(u, v): 0 \leqq u \leqq 2 \pi,-h \leqq v \leqq h\}$ exactly twice, it follows that

$$
E_{2}=\frac{2}{\pi} \int_{0}^{h} \int_{0}^{2 \pi}\left(\log ^{+}\left|\sum_{d_{n}<s} K\left(r, d_{n}, u+i v\right)\right|\right)\left(h^{2}-v^{2}\right)^{-1 / 2} d u d v .
$$

We define

$$
\varepsilon=\varepsilon(r)=\min \left\{\exp \left(-T\left(c^{2}(r), f\right)\right),(1-r)^{5}\right\},
$$

and

$$
\begin{aligned}
D=D(\varepsilon)= & \bigcup_{d_{n}<s}\left\{\left(\log \left(d_{n} r^{-1}\right)-\varepsilon, \log \left(d_{n} r^{-1}\right)+\varepsilon\right)\right. \\
& \left.\cup\left(-\log \left(d_{n} r^{-1}\right)-\varepsilon,-\log \left(d_{n} r^{-1}\right)+\varepsilon\right)\right\} .
\end{aligned}
$$

We will evaluate the integral in (2.14) over values in $[0, h]-D$ and then over $v$ values in $D \cap[0, h]$. We begin by obtaining a lower bound for the denominator of $\left|K\left(r, d_{n}, v+i v\right)\right|$ (see (2.3)). If $r^{2}+$ $d_{n}^{2}-2 r d_{n} \cos \left(u_{0}+i v_{0}\right)=0$ for $\left|v_{0}\right| \leqq h$, then

$$
\begin{aligned}
r^{2} & +d_{n}^{2}-2 r d_{n} \cos (u+i v) \\
& =r^{2}+d_{n}^{2}-2 r d_{n} \cos (u+i v)-\left(r^{2}+d_{n}^{2}-2 r d_{n} \cos \left(u_{0}+i v_{0}\right)\right) \\
& =-2 r d_{n}\left(\cos (u+i v)-\cos \left(u_{0}+i v_{0}\right)\right) \\
& =4 r d_{n} \sin \left(\frac{1}{2}\left(u-u_{0}\right)+\frac{i}{2}\left(v-v_{0}\right)\right) \sin \left(\frac{1}{2}\left(u+u_{0}\right)+\frac{i}{2}\left(v+v_{0}\right)\right) .
\end{aligned}
$$

There is an absolute constant $B$ so that $|\sin z| /|\operatorname{Im} z|>B$. If $v \notin D$, then $\left|v \pm v_{0}\right|>\varepsilon$ and $\left|\sin \left(\left(u \pm u_{0}\right) / 2+i\left(v \pm v_{0}\right) / 2\right)\right|>B\left|v \pm v_{0}\right|>B \varepsilon$. Hence, for $v \notin D, d_{n} \neq 0$ and $r>R$, the denominator of $\mid K\left(r, d_{n}\right.$, $u+i v) \mid$ is

$$
\left|r^{2}+d_{n}^{2}-2 r d_{n} \cos (u+i v)\right|>4 r d_{n} B^{2} \varepsilon^{2} .
$$

Also, since $|v| \leqq h$ and $\cos (u+i v)=\cos u \cosh v-i \sin u \sinh v$, we have that the numerator of $\left|K\left(r, d_{n}, u+i v\right)\right|$ is

$$
\left|\boldsymbol{r}^{2}-r d_{n} \cos (u+i v)\right| \leqq 1+\cosh v+\sinh |v|<4 .
$$


Thus, since $K(r, 0, u+i v)=1$ and $\int_{0}^{h}\left(h^{2}-v^{2}\right)^{-1 / 2} d v=\pi / 2$, we have for $d_{0}=\min \left\{d_{k} \neq 0: k=1,2,3, \cdots\right\}$, and for $r>R$

$$
\begin{aligned}
\int_{[0, h]-D} & \frac{1}{2 \pi} \int_{0}^{2 \pi}\left(\log ^{+}\left|\sum_{d_{n}<s} K\left(r, d_{n}, u+i v\right)\right|\right)\left(h^{2}-v^{2}\right)^{-1 / 2} d u d v \\
& \leqq \int_{0}^{h}\left(\log \left(n\left(s, f^{\prime}\right)+n\left(s, \frac{1}{f^{\prime}}\right)\right)-A\left(d_{0}\right) \log \varepsilon\right)\left(h^{2}-v^{2}\right)^{-1 / 2} d v \\
& <A\left(\alpha, d_{0}\right)(T(c(s), f)-\log (1-r)) .
\end{aligned}
$$

Furthermore, since $\int_{0}^{2 \pi}|\log | c-\left.\cos t\right|^{-1} \mid d t<A$ for all real $c$, and a straight forward calculation yield that for all $d_{n} \neq 0$,

$$
\begin{aligned}
\int_{0}^{2 \pi} \log ^{+} \mid K & \left(r, d_{n}, u+i v\right) \mid d u \\
& =\int_{0}^{2 \pi} \log ^{+}\left|\frac{r^{2}-r d_{n} \cos (u+i v)}{r^{2}+d_{n}^{2}-2 r d_{n} \cos (u+i v)}\right| d u \\
& <8 \pi+\left|\log \left(2 r d_{0}\right)\right|+\int_{0}^{2 \pi} \log ^{+}\left|\left(r^{2}+d_{n}^{2}\right)\left(2 r d_{n}\right)^{-1}-\cos u\right|^{-1} d u \\
& <8 \pi+\left|\log \left(2 r d_{0}\right)\right|+A=A\left(d_{0}\right) .
\end{aligned}
$$

Hence, using Lemma 1.6, for $r>R$

$$
\begin{aligned}
\int_{0}^{2 \pi} \log ^{+} & \left|\sum_{d_{n}<s} K\left(r, d_{n}, u+i v\right)\right| d u \\
\leqq & 2 \pi \log \left(n\left(s, f^{\prime}\right)+n\left(s, \frac{1}{f^{\prime}}\right)\right) \\
& \quad+\sum_{d_{n}<s} \int_{0}^{2 \pi} \log ^{+}\left|K\left(r, d_{n}, u+i v\right)\right| d u \\
\leqq & 2 \pi \log \left(n\left(s, f^{\prime}\right)+n\left(s, \frac{1}{f^{\prime}}\right)\right)+A\left(d_{0}\right)\left(n\left(s, f^{\prime}\right)+n\left(s, \frac{1}{f^{\prime}}\right)\right) \\
< & A\left(\alpha, d_{0}\right)(1-r)^{-1}(T(c(s), f)-\log (1-r)) .
\end{aligned}
$$

The measure of $D$ is no more than $\delta=\delta(\varepsilon)=2\left(n\left(s, f^{\prime}\right)+n\left(s, 1 / f^{\prime}\right)\right) \varepsilon$. Also,

$$
\begin{aligned}
\int_{D \cap[0, h]}\left(h^{2}-v^{2}\right)^{-1 / 2} d v & \leqq \int_{h-\delta}^{h}\left(h^{2}-v^{2}\right)^{-1 / 2} d v=\sin ^{-1}(1)-\sin ^{-1}\left(1-\delta h^{-1}\right) \\
& =\frac{\pi}{2}-y
\end{aligned}
$$

where $y=\sin ^{-1}\left(1-\delta h^{-1}\right)$. Since $\lim _{w \rightarrow \pi / 2}(\sin \pi / 2-\sin w) /(\pi / 2-w)^{2}=$ $1 / 2$, we have for $r>R$

$$
\frac{\pi}{2}-y \leqq 2\left(\sin \frac{\pi}{2}-\sin y\right)^{1 / 2}=2\left(1-\left(1-\delta h^{-1}\right)\right)^{1 / 2}=\left(4 \delta h^{-1}\right)^{1 / 2} .
$$


Therefore,

$$
\int_{D \cap[0, h]}\left(h^{2}-v^{2}\right)^{-1 / 2} d v \leqq\left(4 \delta h^{-1}\right)^{1 / 2}=\left(8 h^{-1}\left(n\left(s, f^{\prime}\right)+n\left(s, \frac{1}{f^{\prime}}\right)\right) \varepsilon\right)^{1 / 2}
$$

and from (2.19) and Lemma 1.6,

$$
\begin{aligned}
& \int_{D \cap[0, h]} \int_{0}^{2 \pi}\left(\log ^{+}\left|\sum_{d_{n}<s} K\left(r, d_{n}, u+i v\right)\right|\right)\left(h^{2}-v^{2}\right)^{-1 / 2} d u d v \\
& \leqq A\left(\alpha, d_{0}\right)(1-r)^{-1}(T(c(s), f)-\log (1-r)) \\
& \times\left(8 h^{-1}\left(n\left(s, f^{\prime}\right)+n\left(s, \frac{1}{f^{\prime}}\right)\right) \varepsilon\right)^{1 / 2} \\
& \leqq A\left(\alpha, d_{0}\right)(1-r)^{-2}(T(c(s), f)-\log (1-r))^{3 / 2} \varepsilon^{1 / 2}=o(1)
\end{aligned}
$$

by the definition of $\varepsilon$ (see (2.15)). From (2.14), (2.18) and (2.20) we conclude that for $r>R$

$$
E_{2}<A(\alpha, f)(T(\mathcal{c}(s), f)-\log (1-r)) .
$$

Since $s=c(r)$ it follows from (2.10), (2.13) and (2.21) that for $r>R$ and for some constant $A=A(\alpha, f)$

$$
L_{3}<A(\alpha, f)\left(T\left(c^{2}(r), f\right)-\log (1-r)\right) .
$$

Finally, we conclude from (2.7) and Lemmas 2.2, 2.3, 2.4 and 2.5 for $r \notin \Delta, r>R$ and for some constant $A=A(\alpha, f)$

$$
2 h \phi\left(r, z f^{\prime \prime}(z) / f^{\prime}(z)+1\right)<A\left(T\left(c^{2}(r), f\right)-\log (1-r)\right) .
$$

Part (i) of the theorem now follows from Lemma 1.4 since $h=$ $(1-\alpha)(1-r) / 2$, and $c^{2}(r)=c_{0}(r)$.

3. Proof of part (ii) of the theorem. We have obtained an upper bound for $\phi(r, f)$ off an exceptional set of $r$ values, but the techniques used in $\S 2$ do not yield any upper bound for $\phi(r, f)$ on the exceptional set. In this section we obtain an upper bound for $\phi(r, f)$ on the exceptional set by bounding $\phi\left(r, z f^{\prime \prime} \mid f^{\prime}+1\right)$. This upper bound for $\phi(r, f)$ will yield, upon integration, the appropriate bound for $\Phi(r, f)$.

We let $c(r)=(1-\gamma)+\gamma r$ with $\gamma$ as in Lemma 1.2. By Lemma 1.2 we can write $z f^{\prime \prime}(z) / f^{\prime}(z)+1=g_{1}(z) / g_{2}(z)$ where $g_{1}$ and $g_{2}$ are holomorphic in the unit disk and for $r>R$

$$
\begin{aligned}
\max \left(T\left(r, g_{1}\right), T\left(r, g_{2}\right)\right) & <A(1-r)^{-p} T\left(c(r), z f^{\prime \prime} z / f^{\prime}(z)+1\right) \\
& <A(1-r)^{-p}(T(c(r), f)-\log (1-r))
\end{aligned}
$$

where $p$ is a positive integer and we have used Lemma 1.6 and well known properties of the characteristic function. 
We have $\operatorname{Re}\left(z f^{\prime \prime}(z) / f^{\prime}(z)+1\right)=\operatorname{Re}\left(g_{1}(z) \overline{\left.g_{2}(z)\right)} /\left|g_{2}(z)\right|^{2}\right.$. We let $u_{j, r}(\theta)=$ $\operatorname{Re} g_{j}\left(r e^{i \theta}\right)$ and $v_{j, r}(\theta)=\operatorname{Im} g_{j}\left(r e^{i \theta}\right)$ for $j=1,2$ and define $J_{r}$ by

$$
\begin{aligned}
J_{r}(\theta) & =\operatorname{Re}\left(g_{1}\left(r e^{i \theta}\right) \overline{g_{2}\left(r e^{i \theta}\right)}\right)=\left|g_{2}\left(r e^{i \theta}\right)\right|^{2} \operatorname{Re}\left(\left(r e^{i \theta} f^{\prime \prime}\left(r e^{i \theta}\right) / f^{\prime}\left(r e^{i \theta}\right)\right)+1\right) \\
& =u_{1, r}(\theta) u_{2, r}(\theta)+v_{1, r}(\theta) v_{2, r}(\theta) .
\end{aligned}
$$

Now choose $r_{0}>0$ so that (3.1), Lemma 3.3, (3.8) and (3.12) of this section hold for $r>r_{0}$. For $\gamma$ as in Lemma 1.2 let

$$
c_{0}(r)=\left(1-\gamma^{1 / 4}\right)+\gamma^{1 / 4} r \text { and } s_{n}=c_{0}^{n}(r) .
$$

We note that if we let $s_{0}=r_{0}$ then $c_{0}^{4}(r)=c(r)$ and $\bigcup_{n=0}^{\infty}\left[s_{n}, s_{n+1}\right)=$ $\left[r_{0}, 1\right)$.

LEMmA 3.1. If $r \in\left[s_{n}, s_{n+1}\right), f\left(r e^{i \theta}\right) \neq 0$ for $0 \leqq \theta \leqq 2 \pi$, and the distance from $|z|=r$ to the nearest zero of $g_{2}(z)$ is no less than $\eta r$, where $\eta<\eta_{0}<1$, then there is a $\theta_{0} \in[0,2 \pi)$ such that

$$
\log \left|J_{r}\left(\theta_{0}\right)\right|>A\left(s_{n+2}-s_{n+1}\right)^{-2}\left(T\left(c\left(s_{n+2}\right), f\right)-\log \left(1-s_{n+2}\right)\right) \log \eta .
$$

Proof. Applying Lemma 1.1 to $g_{2}(z) /\left|g_{2}(0)\right|$ or $g_{2}(z) / c_{k} z^{k}$ for appropriate $k$ and $c_{k}$ in $|z| \leqq s_{n+2}$, we obtain a union of disks $C\left(s_{n}, \eta\right)$, centered at the zeros of $g_{2}$ in $0<|z| \leqq s_{n+2}$, the sum of whose radii does not exceed $\eta s_{n+1}$, such that in $\left\{r_{0} \leqq|z| \leqq s_{n+1}\right\}-C\left(s_{n}, \eta\right)$

(3.4) $\log \left|g_{2}(z)\right|>A\left(s_{n+2}-s_{n+1}\right)^{-2} T\left(s_{n+2}, g\right) \log \eta$

$$
>A\left(s_{n+2}-s_{n+1}\right)^{-2}\left(T\left(c\left(s_{n+2}\right), f\right)-\log \left(1-s_{n+2}\right)\right) \log \eta .
$$

We let $B\left(s_{n}, \eta\right)=\left\{r: f\left(r e^{i \theta}\right) \in C\left(s_{n}, \eta\right)\right.$ for some $\left.0 \leqq \theta<2 \pi\right\}$, and

(3.5) $E\left(s_{n}, \eta\right)=\left[s_{n}, s_{n+1}\right) \cap\left\{B\left(s_{n}, \eta\right) \cup\{r: f\right.$ has a zero of modulus $\left.r\}\right\}$. If $r \in\left[s_{n}, s_{n+1}\right)-E\left(s_{n}, \eta\right)$, then $g_{1}(z) / g_{2}(z)$ has no poles (and hence $f$ has no zeros or poles) on $|z|=r$. Thus $\omega=f\left(r e^{i \theta}\right), 0 \leqq \theta \leqq 2 \pi$ is a closed path in the plane and by (1.1)

$$
\frac{1}{2 \pi} \int_{0}^{2 \pi}\left|\operatorname{Re}\left(r e^{i \theta} f^{\prime \prime}\left(r e^{i \theta}\right) / f^{\prime}\left(r e^{i \theta}\right)\right)+1\right| d \theta \geqq 1 .
$$

Consequently, there is a $\theta_{0} \in[0,2 \pi)$ such that

$$
\left|\operatorname{Re}\left(r e^{i \theta} f^{\prime \prime}\left(r e^{i \theta}\right) / f^{\prime}\left(r e^{i \theta}\right)\right)+1\right| \geqq 1,
$$

which together with (3.2) and (3.4) yields the lemma.

Lemma 3.2. If $r \in\left[s_{n}, s_{n+1}\right)$ and $\theta$ is complex, then $H_{r}(\theta)$ is holomorphic in $|\operatorname{Im} \theta|<-\log r$ and for $|\operatorname{Im} \theta| \leqq \log \left(c\left(s_{n+1}\right) / s_{n+1}\right)$ we have for some positive integer $p$, 


$$
\begin{aligned}
\left|J_{r}(\theta)\right|< & \left(s_{n+3}-s_{n+2}\right)^{-1 / 2} \exp \left\{A\left(s_{n+4}-s_{n+3}\right)^{-(p+1)}\right. \\
& \left.\times\left[T\left(c\left(s_{n+4}\right), f\right)-\log \left(1-s_{n+4}\right)\right]\right\} .
\end{aligned}
$$

Proof. If $g_{1}(z)=\sum_{n=0}^{\infty} a_{n} z^{n}$ where $a_{n}=\alpha_{n}+i \beta_{n}, \alpha_{n}, \beta_{n}$ real, then let $g_{1}^{*}(z)=\sum_{n=0}^{\infty}\left|a_{n}\right| z^{n}$. We note that by Lemma 4 of [10]

$$
M\left(r, g_{1}^{*}\right)<(R-r) M(R, g)
$$

for $0<r<R<1$. Also, for real $\theta$

$$
u_{1, r}(\theta)=\sum_{n=0}^{\infty}\left(\alpha_{n} \cos n \theta-\beta_{n} \sin n \theta\right) r^{n} .
$$

If we let $\theta$ be complex, (3.6) implies that $u_{1, r}(\theta)$ is holomorphic in $|\operatorname{Im} \theta|<-\log r$. If $|\operatorname{Im} \theta|<\log \left(s_{n+2} / s_{n+1}\right)<-\log r$, then

$$
\begin{aligned}
\left|u_{1, r}(\theta)\right| \leqq & 2 \sum_{n=0}^{\infty}\left|a_{n}\right|\left(r \exp \left(\log \left(s_{n+2} / s_{n+1}\right)\right)\right)^{n} \leqq 2 g_{1}^{*}\left(s_{n+2}\right) \\
\leqq & 2 M\left(s_{n+2}, g_{1}^{*}\right)<2\left(s_{n+3}-s_{n+2}\right)^{-1 / 2} M\left(s_{n+3}, g_{1}\right) \\
< & 2\left(s_{n+3}-s_{n+2}\right)^{-1 / 2} \exp \left\{2\left(s_{n+4}-s_{n+3}\right)^{-1} T\left(s_{n+4}, g_{1}\right)\right\} \\
< & 2\left(s_{n+3}-s_{n+2}\right)^{-1 / 2} \exp \left\{A\left(s_{n+4}-s_{n+3}\right)^{-(p+1)}\right. \\
& \left.\times\left[T\left(c\left(s_{n+4}\right), f\right)-\log \left(1-s_{n+4}\right)\right]\right\},
\end{aligned}
$$

where $p$ is a positive integer and we have used Lemma 1.2 and a well known relationship between $\log ^{+} M(r, f)$ and $T(r, f)$, see [4, p. 18]. Identical statements can be made for $v_{1, r}(\theta), u_{2, r}(\theta)$ and $v_{2, r}(\theta)$ and the lemma follows.

Now choose a positive integer $q$ so that

$$
\frac{1}{2} \log \left(s_{n+2} / s_{n+1}\right) \leqq \pi(2 q)^{-1}<\log \left(s_{n+2} / s_{n+1}\right),
$$

which can always be done provided $r_{0}$ is sufficiently large. If $U_{1}=$ $\left\{\theta:|\operatorname{Im} \theta|<\pi(2 q)^{-1}\right\}$, then $f_{1}(z)=e^{z}$ is a one-to-one transformation of $U_{1}$ onto $U_{2}=\left\{\theta \neq 0:|\arg \theta|<\pi(2 q)^{-1}\right\}$, and $f_{2}(z)=z^{q}$ is a one-to-one transformation of $U_{2}$ onto $U_{3}=\{\theta \neq 0:|\arg \theta|<\pi / 2\}$. Also, $f_{3}(z)=$ $\left(z-e^{\theta_{i} q}\right) /\left(z+e^{\theta_{i} q}\right)$ is a one-to-one transformation of $U_{3}$ onto the unit disk, satisfying $f_{3}\left(e^{\theta_{0} q}\right)=0$, where $\theta_{0}$ is as in Lemma 3.1. If we let $L^{-1}(z)=f_{3}\left(f_{2}\left(f_{1}(z)\right)\right)$, then $L(z)$ is a one-to-one transformation of the unit disk onto $U_{1}$, satisfying $L(0)=\theta_{0}$. We let $p(q)=\left(e^{\pi q}-1\right) /\left(e^{\pi q}+1\right)$. Elementary calculations show that $L$ maps $\{|w|<p(q)\}$ onto a region in $U_{1}$ containing the interval $\left[\theta_{0}-\pi, \theta_{0}+\pi\right]$ on the real $\theta$-axis. We will use $L$ to prove

LEMma 3.3. If $r \in\left[s_{n}, s_{n+1}\right)-E\left(s_{n}, \eta\right)$, then

$$
\phi(r, f)<\exp \left\{A\left(s_{n+2}-s_{n+1}\right)^{-1}\right\}\left[T\left(c\left(s_{n+4}\right), f\right)-\log \left(1-s_{n+4}\right)\right] \log \frac{1}{\eta}
$$

provided $r>R$. 
Proof. We let $n_{r}(t)$ be the number of zeros of $J_{r}(L(\omega))$ in $|\omega| \leqq t$. Since $J_{r}(L(\omega))$ is holomorphic in $|\omega|<1$, we apply Jensen's theorem to $J_{r} \circ L$ to obtain

$$
\int_{0}^{t} n_{r}(x) x^{-1} d x=-\log \left|J_{r}(L(0))\right|+\frac{1}{2 \pi} \int_{0}^{2 \pi} \log \left|J_{r}\left(L\left(t e^{i \zeta}\right)\right)\right| d \zeta
$$

For $t>p(q)$ we have

$$
\int_{0}^{t} n_{r}(x) x^{-1} d x>n_{r}(p(q)) \log \left(t(p(q))^{-1}\right) .
$$

We note that $-\log p(q)>\exp (-\pi q)$ for sufficiently large $q$, and $q$ will be large enough if $s_{n}$ (or, equivalently, $r_{0}$ ) is large enough. Also, from the definition of $q$, we have $\exp (\pi q)<\exp \left(A\left(s_{n+2}-s_{n+1}\right)^{-1}\right)$. This observation together with (3.7), (3.8), Lemma 3.1 and Lemma 3.2 yield, upon letting $t$ approach 1 ,

$$
\begin{aligned}
n_{r}(p(q))< & {\left[\log \left(t(p(q))^{-1}\right)\right]^{-1} \int_{0}^{t} n_{r}(x) x^{-1} d x } \\
= & {\left[\log \left(t(p(q))^{-1}\right)\right]^{-1}\left\{-\log \left|J_{r}\left(\theta_{0}\right)\right|+\frac{1}{2 \pi} \int_{0}^{2 \pi} \log \left|J_{r}\left(L\left(t e^{i \zeta}\right)\right)\right| d \zeta\right\} } \\
< & \exp \left(A\left(s_{n+2}-s_{n+1}\right)^{-1}\right)\left\{A ( s _ { n + 2 } - s _ { n + 1 } ) ^ { - 2 } \left[T\left(c\left(s_{n+2}\right), f\right)\right.\right. \\
& \left.-\log \left(1-s_{n+2}\right)\right] \log \frac{1}{\eta}-\frac{1}{2} \log \left(s_{n+3}-s_{n+4}\right) \\
& \left.+A\left(s_{n+4}-s_{n+3}\right)^{-(p+1)}\left[T\left(c\left(s_{n+4}\right), f\right)-\log \left(1-s_{n+4}\right)\right]\right\} \\
< & \exp \left(A\left(s_{n+2}-s_{n+1}\right)^{-1}\right)\left[T\left(c\left(s_{n+4}\right), f\right)-\log \left(1-s_{n+4}\right)\right] \log \frac{1}{\eta}
\end{aligned}
$$

Since the zeros of $J_{r}(L(\omega))$ in $|\omega|<p(q)$ include the zeros of $\operatorname{Re}\left(r e^{i \theta} f^{\prime \prime}\left(r e^{i \theta}\right) / f^{\prime}\left(r e^{i \theta}\right)+1\right)$ in the interval $\left[\theta_{0}-\pi, \theta_{0}+\pi\right]$, the lemma follows from Lemma 1.4 .

Let $A_{0}$ be the constant in Lemma 3.3, and let $\delta_{n}=\exp \left(-3 T\left(c\left(s_{n+4}\right)\right.\right.$, $\left.f)-4 A_{0}\left(s_{n+2}-s_{n+1}\right)^{-1}\right)$. Define $E=\bigcup_{n=0}^{\infty} E\left(s_{n}, \delta_{n}\right)$, where $s_{n}$ and $E\left(s_{n}, \delta_{n}\right)$ are defined by (3.3) and (3.5), respectively. Let $\Delta^{\prime}$ be the set in Lemma 1.5 corresponding to $\alpha_{2}=\gamma^{2}$ and $k(r)=B(1-r)^{-1}$ with $B$ a sufficiently large constant to be specified in (3.12) below. Finally, let $P_{1}=\left[0, r_{0}\right], P_{2}=\Delta^{\prime} \cap E$, and $P_{3}=\left(\Delta^{\prime}-E\right) \cap\left[r_{0}, 1\right)$. We will bound

$$
\int_{P_{j}} \dot{\phi}(t, f)(1-t)^{-1} d t \quad \text { for } \quad j=1,2,3 .
$$

If $D(n)=\left\{r<s_{n+2}: g_{2}\right.$ has a zero of modulus $\left.r\right\}$, and if $r_{1} \in D(n)$ then by Lemma 3.3, for $s_{n}>R$ 


$$
\begin{aligned}
& \int_{\max \left(r_{1}-\hat{o}_{n} \cdot s_{n}\right)}^{\min \left(r_{1}+\hat{\delta}_{n} s_{n+1}\right)} \phi(t, f)(1-t)^{-1} d t \\
& \quad<\exp \left\{A_{0}\left(s_{n+2}-s_{n+1}\right)^{-1}\right\}\left(T\left(c\left(s_{n+4}\right), f\right)-\log \left(1-s_{n+4}\right)\right) \\
& \quad \quad \times \int_{r_{1}-\hat{o}_{\eta}}^{r_{1}+\tilde{o}_{\eta}}\left(-\log \left|t-r_{1}\right|\right) d t \\
& \quad<2 \exp \left\{A_{0}\left(s_{n+2}-s_{n+1}\right)^{-1}\right\}\left(T\left(c\left(s_{n+4}\right), f\right)-\log \left(1-s_{n+4}\right)\right)\left(\delta_{n}-\delta_{n} \log \delta_{n}\right) \\
& \quad<\exp \left\{-2 T\left(c\left(s_{n+4}\right), f\right)-2 A_{0}\left(s_{n+2}-s_{n+1}\right)^{-1}\right\} .
\end{aligned}
$$

Since $E\left(s_{n}, \delta_{n}\right) \subset \bigcup_{r \in D(n)}\left(r-\delta_{\eta}, r+\delta_{\eta}\right) \cup\{r: f$ has a zero of modulus $r$, and $g_{2}$ has no more than $n\left(s_{n+2}, g_{2}\right)$ zeros in $|z|<s_{n+2}$, we have from Lemma 1.6 and (3.9) for $r>R$

$$
\begin{aligned}
\int_{E\left(s_{n}, \delta_{n}\right)} \phi(t, f)(1-t)^{-1} d t \\
\quad<\exp \left\{-2 T\left(c\left(s_{n+4}\right), f\right)-2 A_{0}\left(s_{n+2}-s_{n+1}\right)^{-1}\right\} n\left(s_{n+2}, g\right) \\
\quad<1-s_{n}=\gamma^{n / 4}\left(1-r_{0}\right) .
\end{aligned}
$$

Since $E=\bigcup_{n=0}^{\infty} E\left(s_{n}, \delta_{n}\right)$, an elementary calculation shows

$$
\int_{P_{2}} \phi(t, f)(1-t)^{-1} d t<\infty \text {. }
$$

It follows from [10, paragraph after (2.16)] that

$$
\int_{P_{1}} \phi(t, f)(1-t)^{-1} d t<\infty \text {. }
$$

If $r \in\left(\Delta^{\prime}-E\right) \cap\left[s_{n}, s_{n+1}\right)$, then from Lemma 3.3, for $r_{0}>R$

$$
\begin{aligned}
\dot{\phi}( & r, f)(1-r)^{-1} \\
\quad< & (1-r)^{-1} \exp \left\{A_{0}\left(s_{n+2}-s_{n+1}\right)^{-1}\right\}\left[T\left(c\left(s_{n+4}\right), f\right)-\log \left(1-s_{n+4}\right)\right] \\
& \times\left[3 T\left(c\left(s_{n+4}\right), f\right)+4 A_{0}\left(s_{n+2}-s_{n+1}\right)^{-1}\right] \\
< & \exp \left\{2 A_{0}\left(s_{n+2}-s_{n+1}\right)^{-1}\right\} T^{2}\left(c\left(s_{n+4}\right), f\right) \\
< & \exp \left\{B(1-r)^{-1}\right\} T^{2}\left(c\left(c_{0}^{4}(r)\right), f\right) \\
= & \exp \left\{B(1-r)^{-1}\right\} T^{2}\left(c^{2}(r), f\right) \\
\quad< & \exp \left\{T\left(c^{2}(r), f\right)+B(1-r)^{-1}\right\}
\end{aligned}
$$

where $B$ is a constant and we have used the fact that $c_{0}^{4}(r)=c(r)$. Thus, by Lemma 1.5 we have

$$
\int_{P_{3}} \phi(t, f)(1-t)^{-1} d t<\infty .
$$

Finally, we note that the proof of part (i) of the theorem may be altered using Lemma 1.5 with $\Delta^{\prime}$ corresponding to $k(r)=B(1-r)^{-1}$ ( $B$ as in (3.12)) and $\alpha_{2}=\gamma^{2}$ to yield that for $r \notin \Delta^{\prime}$ and $r>R$

$$
\phi(r, f)<A(1-r)^{-1}\left[T\left(c^{2}(r), f\right)+(1-r)^{-1}\right] .
$$


From (3.10), (3.11), (3.13) and (3.14) we conclude for $r>r_{0}$,

$$
\begin{aligned}
\int_{0}^{r} \phi(t, f)(1-t)^{-1} d t & <\int_{0}^{r} A(1-t)^{-2}\left[T\left(c^{2}(t), f\right)+(1-t)^{-1}\right] d t+O(1) \\
& <A\left[T\left(c^{2}(r), f\right)+(1-r)^{-1}\right]\left((1-r)^{-1}-1\right)+O(1) \\
& <A(1-r)^{-1}\left[T\left(c^{2}(r), f\right)+(1-r)^{-1}\right] .
\end{aligned}
$$

The proof of part (ii) of the theorem follows by letting $\alpha_{1}=\gamma^{2}$.

4. Examples. We first give an example to show that $\phi(r, f)$ may equal $O(1)$, and that $\Phi(r, f)$ may equal $O(-\log (1-r))$, for functions of arbitrarily large order. For $\lambda>0$, let

$$
f(z)=\exp \left\{((1+z) /(1-z))^{\lambda}\right\},
$$

where the branch is chosen so that $f(0)=e$. Note that $|f(z)|=1$ implies $\operatorname{Re}\left\{((1+z) /(1-z))^{\lambda}\right\}=0$. Since $(1+z) /(1-z)$ takes $|z|=r$ onto a circle in the right half plane, $\left|\arg ((1+z) /(1-z))^{2}\right|<\pi \lambda / 2$. Also, for $k=0, \pm 1, \pm 2, \cdots, \pm[\lambda / 2],-[\lambda / 2]-1, \arg ((1+z) /(1-z))^{\lambda}=$ $(k+1 / 2) \pi$ if and only if $\arg ((1+z) /(1-z))=1 / \lambda(k+1 / 2) \pi$. For each such $k$, the latter equality holds at most twice on $|z|=r$. Thus, $|f(z)|=1$ at no more than $4([\lambda / 2]+1) \leqq 2 \lambda+4$ points on $|z|=r$. If $L(z)$ is a linear fractional transformation taking $|z|=1$ onto the imaginary axis, and if $g(z)=L(f(z))$, then $\phi(r, g) \leqq 2 \lambda+4$ and $\Phi(r, g) \leqq(2 \lambda+4) \log (1-r)^{-1}$. The order of $g$ can be made arbitrarily large by choosing $\lambda$ sufficiently large.

Now we give an example to show that the factor $(1-r)^{-1}$ in (i) and (ii) of the theorem cannot be replaced by any function $b(r)$ satisfying $b(r)=o\left((1-r)^{-1}\right)$. We use the Lindelöf functions. If $q$ is a positive integer and $q \leqq \lambda \leqq q+1$, then we let

$$
f(z, \lambda)=\prod_{k=1}^{\infty}\left(1-z a_{n}^{-1}\right) \exp \left\{\left(z a_{n}^{-1}\right)+\frac{1}{2}\left(z a_{n}^{-1}\right)^{2}+\cdots+\frac{1}{q}\left(z a_{n}^{-1}\right)^{q}\right\},
$$

where $a_{n}=n^{1 / \lambda}$. It is known [11, p. 18] that $f(z, \lambda)$ has order $\lambda$ and mean type 1 . Thus, for $\varepsilon>0$ and $|z|>R(\varepsilon)$, we have

$$
\log |f(z, \lambda)|<(1+\varepsilon)|z|^{\lambda} \text {. }
$$

We let $g(z, \lambda)=f((1+z) /(1-z), \lambda)$. Thus, for $|(1+z) /(1-z)|>R(\varepsilon)$, (4.1) implies

$$
\log |g(z, \lambda)|<(1+\varepsilon)|(1+z) /(1-z)|^{\lambda} .
$$

Also, there is a constant $K(\varepsilon)$ so that, if $|(1+z) /(1-z)| \leqq R(\varepsilon)$, then

$$
\log |g(z, \lambda)|<K(\varepsilon) .
$$

Since $(1+\varepsilon)\left(\left|1+r e^{i \theta}\right| /\left|1-r e^{i \theta}\right|\right)^{\lambda}=(1+\varepsilon)\left|1+r e^{i \theta}\right|^{2}\left(\left|1-r e^{i \theta}\right|^{2}\right)^{-\lambda / 2} \leqq$ 
$(1+\varepsilon) 2^{\lambda}\left(1-2 r \cos \theta+r^{2}\right)^{-\lambda / 2}$, we have from (4.2) and (4.3)

$$
\begin{aligned}
m(r, g) & =\frac{1}{2 \pi} \int_{-\pi}^{\pi} \log ^{+}\left|g\left(r e^{i \theta}\right)\right| d \theta \\
& \leqq \frac{2^{\lambda}(1+\varepsilon)}{2 \pi} \int_{-\pi}^{\pi}\left(1-2 r \cos \theta+r^{2}\right)^{-(\lambda / 2)} d \theta+K(\varepsilon) .
\end{aligned}
$$

By $\left[2\right.$, p. 65], the latter integral in $(4.4)$ equals $O\left((1-r)^{-(\lambda-1)}\right)$. Thus

$$
T(r, g)=m(r, g)=O\left((1-r)^{-(\lambda-1)}\right) .
$$

Since the image of $|z| \leqq r$ under $(1+z) /(1-z)$ contains the interval $[(1-r) /(1+r),(1+r) /(1-r)]$ on the real $\theta$-axis, we have $n(r, 1 / g) \geqq$ $(1-r)^{-\lambda}$, for $r>R$. By the argument principle, if $f(z) \neq 0$ on $|z|=r$ and $r<R$, then

$$
\phi(r, g) \geqq 2(1-r)^{-\lambda} .
$$

From (4.5) and (4.6), it follows that if $f(z) \neq 0$ on $|z|=r$ and if $r>R$,

$$
\begin{aligned}
(1-r)^{-1} T((1-\beta)+\beta r, g) & =O\left[(1-r)^{-1}(1-((1-\beta)+\beta r))^{-(\lambda-1)}\right] \\
& =O\left[\beta^{-(\lambda-1)}(1-r)^{-\lambda}\right] \leqq A \phi(r, g) .
\end{aligned}
$$

\section{REFERENCES}

1. W. Beck, Efficient Quotient Representations of Meromorphic Functions in the Disk, Thesis, University of Illinois, 1970.

2. P. L. Duren, Theory of $H^{p}$ Spaces, Academic Press, New York and London, 1970.

3. A. Gelfond, Über die Harmonischen Funktionen, Trudy Fiz.-Matem. Inst. Steklova 5 (1934), 148-159.

4. W. K. Hayman, Meromorphic Functions, Oxford Mathematical Monographs, Clarendon Press, Oxford, 1964.

5. S. Hellerstein and J. Korevaar, The real values of an entire function, Bull. Amer. Math. Soc., 70 (1964), 608-610.

6. E. Hille, Analytic Function Theory, Volume II, Ginn and Company, Boston, 1962.

7. B. Ja. Levin, Distribution of Zeros of Entire Functions, Amer. Math. Soc. Transl., Providence, R.I. (1964).

8. J. Miles, Bounds on the ratio $n(r, a) / S(r)$ for meromorphic functions, Trans. Amer. Math. Soc., 162 (1971), 383-393.

9. - Quotient representations of meromorphic functions, J. D'Analyse Mathématique, Vol. XXV (1972), 371-388.

10. J. Miles and D. Townsend, Imaginary values of meromorphic functions, Indiana University Mathematics Journal, 27, No. 3 (1978), 491-503.

11. R. Nevanlinna, Le Théorèm de Picard-Borel et la Théorie des Fontions Meromorphes, Chelsea Publishing Company, Bronx, New York, 1974.

12. D. Townsend, Imaginary Values of Meromorphic Functions, Thesis, University of Illinois, 1976.

Received May 31, 1978 and in revised form May 30, 1980. This work was supported in part by a grant from the Indiana-Purdue University at Fort Wayne Research and Instructional Development Support Program. 


\section{PACIFIC JOURNAL OF MATHEMATICS}

\section{EDITORS}

DONALD BABBITT (Managing Editor)

University of California

Los Angeles, California 90024

Hugo RossI

University of Utah

Salt Lake City, UT 84112

C. C. MOORE and ANDREW OGG

University of California

Berkeley, CA 94720

\section{J. DugundJI}

Department of Mathematics University of Southern California Los Angeles, California 90007

R. FinN and J. Milgram Stanford University Stanford, California 94305

\section{ASSOCIATE EDITORS}
R. ARENS
E. F. BECKENBACH
B. H. Neumann
F. WOLF
K. YoshidA

\section{SUPPORTING INSTITUTIONS}

UNIVERSITY OF ARIZONA

UNIVERSITY OF BRITISH COLUMBIA

CALIFORNIA INSTITUTE OF TECHNOLOGY

UNIVERSITY OF CALIFORNIA

MONTANA STATE UNIVERSITY

UNIVERSITY OF NEVADA, RENO

NEW MEXICO STATE UNIVERSITY

OREGON STATE UNIVERSITY
UNIVERSITY OF OREGON

UNIVERSITY OF SOUTHERN CALIFORNIA

STANFORD UNIVERSITY

UNIVERSITY OF HAWAII

UNIVERSITY OF TOKYO

UNIVERSITY OF UTAH

WASHINGTON STATE UNIVERSITY

UNIVERSITY OF WASHINGTON 


\section{Pacific Journal of Mathematics}

\section{Vol. 96, No. $1 \quad$ November, 1981}

Hédi Amara, Groupe des classes et unité fondamentale des extensions quadratiques relatives à un corps quadratique imaginaire principal $\ldots \ldots \ldots 1$

Douglas S. Bridges, On the isolation of zeroes of an analytic function $\ldots \ldots 13$ Andrew J. Casson and John L. Harer, Some homology lens spaces which

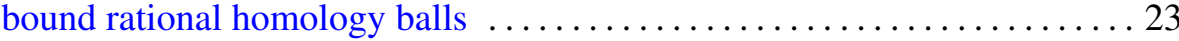

Z. A. Chanturia, On the absolute convergence of Fourier series of the classes $H^{\omega} \cap V[v]$

J.-F. Colombeau and Mário Carvalho Matos, On some spaces of entire functions defined on infinite-dimensional spaces $\ldots \ldots \ldots \ldots \ldots \ldots \ldots 63$

Edwin Duda, Pointwise periodic homeomorphisms on chainable continua . . .77

Richard F. Gustafson, A simple genus one knot with incompressible spanning surfaces of arbitrarily high genus $\ldots \ldots \ldots \ldots \ldots \ldots \ldots \ldots 1$

Fumio Hiai, Masanori Ohya and Makoto Tsukada, Sufficiency, KMS condition and relative entropy in von Neumann algebras

Ted Hurley, Intersections of terms of polycentral series of free groups and free Lie algebras. II .................................. 111

Robert Edward Jamison, II, Partition numbers for trees and ordered sets . . 115 R. D. Ketkar and N. Vanaja, A note on FR-perfect modules ............. 141 Michihiko Kikkawa, On Killing-Ricci forms of Lie triple algebras ....... 153

Jorge Lewowicz, Invariant manifolds for regular points 163

Richard W. Marsh, William H. Mills, Robert L. Ward, Howard Rumsey and Lloyd Richard Welch, Round trinomials .....

Claude Schochet, Topological methods for $C^{*}$-algebras. I. Spectral sequences

Yong Sian So, Polynomial near-fields?

Douglas Wayne Townsend, Imaginary values of meromorphic functions in the disk

Kiyoshi Watanabe, Coverings of a projective algebraic manifold .. 243

Martin Michael Zuckerman, Choosing $l$-element subsets of $n$-element sets 